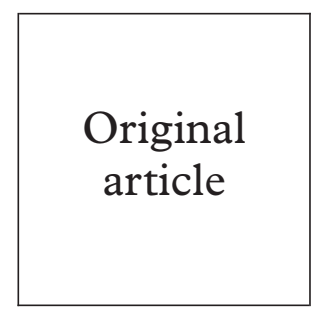

\title{
Assessment of Chlamydia trachomatis prevalence by PCR and LCR in women presenting for termination of pregnancy
}

\author{
Suzanne M Garland, Sepehr Tabrizi, Julene Hallo, Shujun Chen
}

Objectives: To determine the prevalence of Chlamydia trachomatis in a patient population presenting for legal termination of pregnancy by polymerase chain reaction (PCR) and ligase chain reaction (LCR), from first catch urine and self administered tampons, and comparing with the traditionally collected endocervical swab tested by both PCR and culture.

Methods: Consecutive women attending for legal termination of pregnancy were screened for chlamydia by patient collected first catch urine and tampon, and physician collected endocervical swab.

Results: Of 1175 patients with complete samples, there were $33(2.8 \%)$ in whom chlamydia was detected by two or more assays from one or more sample site. Chlamydia was detected equally well by both PCR and LCR in first catch urine $(p=0.25)$, tampon $(p=0.5)$, and endocervical swab $(p=0.5)$. However, both PCR and LCR were significantly better than culture of an endocervical swab $(\mathrm{p}=0.0005)$ for detection of $C$ trachomatis.

Conclusion: The prevalence of chlamydia in patients presenting for termination of pregnancy was $2.8 \%$. A simple efficient way of performing screening for chlamydia for women presenting for termination of pregnancy is by first catch urine or tampon, which can be tested by the highly sensitive amplification assays, PCR or LCR.

(Sex Transm Inf 2000;76:173-176)

Keywords: polymerase chain reaction; ligase chain reaction; Chlamydia trachomatis; pregnancy

\section{Introduction}

Women with Chlamydia trachomatis genital infection are at a significant risk of post-abortal sepsis. ${ }^{12}$ Furthermore, studies have shown that screening and treatment for $C$ trachomatis near the time of the termination, is associated with a decreased risk of pelvic sepsis. ${ }^{3}$ Moreover, it is a recommendation by both the Centres for Disease Control (USA) ${ }^{5}$ and the National Venereology Committee of Australia, ${ }^{6}$ that women presenting for elective abortion be screened for chlamydia.

While diagnosis of chlamydia has traditionally been by culture, rapid antigen tests, or DNA probes, recently the more sensitive nucleic acid amplification assays such as polymerase chain reaction (PCR) and ligase chain reaction (LCR) have been utilised. ${ }^{78}$ Detection of $C$ trachomatis in women by conventional tests has traditionally required vaginal examinations to collect endocervical cells while for males, urethral swabs are necessary. However, with the increased sensitivity of the amplification tests, collection of clinical samples by non-invasive, patient administered measures have been evaluated and shown to perform at least as well. In particular, detection of chlamydia by LCR or PCR in first catch urine in both females and males has been reported to have a high sensitivity and specificity. ${ }^{9}{ }^{10}$ Furthermore, we have shown that self administered tampon specimens from women for subsequent PCR detection of $C$ trachomatis, ${ }^{11}$ Trichomonas vaginalis, ${ }^{12}$ and Neisseria gonorrhoeae have a significantly greater sensitivity than conventional collection and testing procedures. ${ }^{13}$

In this study detection of $C$ trachomatis by PCR and LCR was evaluated in women presenting for termination of pregnancy, by analysing various patient collected samples and comparing the results with endocervical specimens.

\section{Methods}

PATIENT POPULATION

The study population comprised 1245 consecutive patients who attended the pregnancy advisory service, Royal Women's Hospital, Melbourne, Australia over a 12 month period from August 1996 to July 1997, for legal termination of pregnancy. All patients recruited gave informed consent; of those approached only two refused to participate.

\section{SPECIMEN COLLECTION}

Patients were initially asked to collect approximately $10 \mathrm{ml}$ of a first catch urine into a standard sterile urine collection container, followed by a tampon specimen (self inserted and immediately withdrawn) which was placed into a sterile specimen jar containing transport medium (0.14 M NaCl, $3 \mathrm{mM} \mathrm{KCl}, 10 \mathrm{mM}$ $\left.\mathrm{HPO}_{4}, 2 \mathrm{mM} \mathrm{KH} \mathrm{PO}_{4}\right)$. Patients were then assessed by a clinician who performed a pelvic examination including collection of two endocervical samples per patient (that is, for culture and LCR). The ectocervix was first cleaned of any excess mucus using a large cotton swab. The order of the two swabs was randomised throughout the study. Endocervical 
swabs collected for LCR were transported in medium provided by Abbott Diagnostics (Abbott Park, IL, USA). For tissue culture isolation, cotton tipped ENT swabs (Medical Wire and Equipment Company, Wilts) were rotated within the endocervical canal, withdrawn, and placed into SPG transport medium (218 $\mathrm{mM}$ sucrose, $3.8 \mathrm{mM} \mathrm{KH} \mathrm{PO}_{4}, 7.2 \mathrm{mM}$ $\mathrm{K}_{2} \mathrm{HPO}_{4}, 4.9 \mathrm{mM}$ L-glutamate, $150 \mathrm{mg} / \mathrm{ml}$ vancomycin, $10 \mathrm{mg} / \mathrm{ml}$ amphotericin $\mathrm{B}, 20$ $\mathrm{mg} / \mathrm{ml}$ gentamicin, $\mathrm{pH}$ 7) and transported to the laboratory on ice within 14 hours of collection. Specimens were either cultured immediately or frozen at $-70^{\circ} \mathrm{C}$ until processed..$^{14}$ For detection by PCR, an aliquot of the sample collected for culture was utilised.

TESTING

Urine was processed by first collecting $1 \mathrm{ml}$ for LCR then processed as described by the manufacturer, while $10 \mathrm{ml}$ of urine was pelleted for PCR, from which DNA was extracted utilizing Qaigen Kit (Qiagen, Valencia, CA, USA). Tampon specimens were processed as previously described. ${ }^{11}$ For PCR of endocervical swabs, an aliquot of $100 \mu$ from each culture had DNA extracted by the Qiagen system followed by PCR testing.

All first catch urine, tampon samples, and endocervical swabs had PCR and LCR performed for detection of $C$ trachomatis. In addition, the endocervical swabs had standard culture detection for C trachomatis. ${ }^{14}$ The PCR method was directed against the major outer membrane protein (MOMP) of $C$ trachomatis and based on the method of Holland et al, ${ }^{15}$ and details are as previously described. ${ }^{11}$ In addition to PCR amplification for $C$ trachomatis, all specimens were also amplified for a $260 \mathrm{bp}$ region of human $\beta$ globin gene as an internal positive control for each specimen, to ensure that sufficient amplifiable DNA was present in the sample. ${ }^{7}$ Specimen contamination and carry over were prevented by using barriered tips, aliquoting of reagents and performing the various stages of PCR in different rooms. ${ }^{11}$ All LCR assays were performed as directed by the manufacturer (Abbott Diagnostics).

All endocervical swabs were cultured into cycloheximide treated HeLa 229 cells in 48 well multiwell trays. Specimens were stained with fluorescein conjugated antichlamydial monoclonal antibody ("Pathfinder" Kallestad, Chaska, MI, USA) and viewed under fluorescent microscopy on an inverted fluorescent microscope (Leitz, Sydney, Australia) as previously described. ${ }^{14}$

All assays on clinical samples were performed blinded to the results of one another. A patient was considered positive for $C$ trachomatis if the endocervical sample was positive by culture and/or at least one of first catch urine, tampon, or endocervical sample was positive by PCR and LCR.

In order to resolve discrepant results from patients where only one test type or sample type was positive, specimens were subjected to additional testing. For samples negative by culture and positive by PCR or LCR, direct immunofluorescence assay (DFA) was per-
Table 1 Results for $C$ trachomatis detection by sample site and assay

\begin{tabular}{llll}
\hline & \multicolumn{3}{l}{ Positive results } \\
\cline { 2 - 4 } Specimen tested & $P C R$ & LCR & Culture \\
\hline First catch urine & 34 & 31 & $\mathrm{ND}^{\star}$ \\
Tampon & 31 & 29 & $\mathrm{ND}^{\star}$ \\
Endocervical swabs & 27 & 29 & 15 \\
\hline
\end{tabular}

${ }^{\star} \mathrm{ND}=$ not done.

formed on the centrifuged deposit of the culture sample, when sufficient samples were available.

Patients positive for $C$ trachomatis were randomised to receive azithromycin $1 \mathrm{~g}$ orally immediately or doxycycline $100 \mathrm{mg}$ twice daily for 10 days. All patients were invited for follow up (with repeat samples as above) with their sexual partners, 2-4 weeks after the procedure.

\section{STATISTICS}

Paired categorical variables were analysed by a McNemar Q test using a PROPHET software package (National Institute of Health, USA).

\section{Results}

Of the 1245 patients from whom specimens were collected, 70 had an incomplete set of specimens (63 no endocervical swab, six no urine, four no tampon). Of these, four patients were positive for $C$ trachomatis in two or more sites or assays. These four patients had no endocervical swab collected, but had a first catch urine and tampon, of which all were positive by PCR and LCR and therefore deemed positive for $C$ trachomatis and treated accordingly.

Of the 1175 patients with a complete set of samples, 36 patients were positive for $C$ trachomatis on at least one test. Thirty three patients were considered true positives, resulting in an overall prevalence of $2.8 \%$. The breakdown of the 33 positive patients is shown in table 1 . Of the 15 patients with endocervical swabs positive by culture, all their other specimens (first catch urine, tampon, endocervical swab) were also positive by PCR and LCR (except one urine negative by LCR). Ten of the patients with negative endocervical swabs by culture were positive in all other samples, by all other methods. One patient inconclusive on culture was positive by all other tests, from all other samples (the DFA on the culture deposit also had a low number of elementary bodies). A further three patients who were culture negative were also $\beta$ globin negative and negative for $C$ trachomatis on PCR testing; two of these were also LCR negative on the endocervical swab, although all three were positive by PCR and LCR of the first catch urine and tampon. One further patient negative by culture, negative PCR endocervical swab (but LCR positive endocervical swab), was positive by both PCR and LCR for both first catch urine and tampon samples.

There were six patients in whom only one site or test was positive (two were positive by both PCR and LCR on first catch urine, while one other patient was endocervical swab positive by PCR and LCR and all three there- 
Table 2 Comparison of PCR and LCR

\begin{tabular}{|c|c|c|c|c|c|c|c|c|c|}
\hline & \multicolumn{9}{|c|}{$P C R$} \\
\hline & \multicolumn{3}{|c|}{ First catch urine } & \multicolumn{3}{|c|}{ Tampon } & \multicolumn{3}{|c|}{ Endocervical swab } \\
\hline & Pos & $\mathrm{Neg}$ & Total & Pos & $\mathrm{Neg}$ & Total & Pos & $\mathrm{Neg}$ & Total \\
\hline \multicolumn{10}{|l|}{ LCR } \\
\hline Pos & 31 & 0 & 31 & 29 & 0 & 29 & 27 & 2 & 29 \\
\hline Neg & 3 & 1141 & 1144 & 2 & 1144 & 1146 & 0 & 1146 & 1146 \\
\hline Total & 34 & 1141 & 1175 & 31 & 1144 & 1175 & 27 & 1148 & 1175 \\
\hline \multirow[t]{9}{*}{$\mathrm{p}$ Value } & & & 0.25 & & & 0.5 & & & 0.5 \\
\hline & & & \multicolumn{7}{|c|}{$\begin{array}{l}\text { Table } 3 \text { Comparison of culture for endocervical sample to } \\
\text { LCR and PCR }\end{array}$} \\
\hline & & & & \multicolumn{3}{|c|}{$P C R$} & \multicolumn{3}{|c|}{$L C R$} \\
\hline & & & & Pos & $\mathrm{Neg}$ & Total & Pos & $\mathrm{Neg}$ & Total \\
\hline & & & \multicolumn{7}{|l|}{ Culture } \\
\hline & & & Pos & 15 & 0 & 15 & 15 & 0 & 15 \\
\hline & & & Neg & 12 & 1148 & 1160 & 14 & 1146 & 1160 \\
\hline & & & Total & 27 & 1148 & 1175 & 29 & 1146 & 1175 \\
\hline & & & p Value & & & 0.0005 & & & 0.0005 \\
\hline
\end{tabular}

fore were defined as positive patients and treated accordingly). Of the other three patients, in whom only one site by one test was positive, two were positive by PCR first catch urine alone and one positive by PCR on tampon alone; hence all three of these patients were deemed negative.

There were no patients positive by culture alone. Of the 10 culture negative patients, positive by PCR and LCR, four culture pellets were available for DFA and three showed low number of elementary bodies.

Detection of $C$ trachomatis by PCR or LCR from endocervical swab, first catch urine, or tampon was not statistically different $(\mathrm{p}=0.25$, 0.5 , and 0.5 respectively, see table 2 ); whereas from endocervical swab, detection by PCR/ LCR compared with culture was significantly better $(p=0.0005)$ (table 3$)$. The positive predictive value (PPV) and negative predictive value (NPV) of PCR of endocervical swab sample compared with culture was $56 \%$ and $100 \%$ respectively, while for LCR it was $52 \%$ and $100 \%$ respectively. The PPV and NPV of LCR using the expanded gold standard for endocervical samples as a comparison was $93 \%$ and $100 \%$ respectively, whereas for PCR it was $100 \%$ for both. For the tampon or first catch urine comparison against the expanded gold standard, the PPV, and NPV were the same at $100 \%$ and $99.8 \%$ respectively.

Of the 33 patients defined as positive for chlamydia, 28 returned for follow up after treatment and all were also assessed clinically. Of 13 from whom test of cure samples were collected, none was positive for $C$ trachomatis. There was no difference in outcome for those patients treated with doxycycline compared with azithromycin.

\section{Discussion}

The prevalence of $C$ trachomatis in women presenting for termination of pregnancy in a teaching hospital in Melbourne, Australia, was calculated at $2.8 \%$. This is lower than the previously determined rate of $4.9 \%$ at the same hospital in $1986^{1}$ and $2 \%$ in 1990 (S Garland unpublished observation), both of which were made by culture and for the same hospital population. This decreased prevalence of chlamydia is consistent with that for other female populations screened in Melbourne, and has been noted since the safe sex campaign of the late 1980s. At the Melbourne Sexual Health Centre the chlamydia detection rate in women for 1997 was $1.3 \%$ (D Jacobs, personal communication), whereas in 1982 it was $14.6 \%, 19839.1 \%, 19892.7 \%$ as determined by culture. ${ }^{16}$

As most $C$ trachomatis infection is asymptomatic there is a need to screen at risk patients to reduce the morbidity from infection, as well as to decrease the incidence and prevalence of this pathogen in the population at large. ${ }^{5}$ Screening and treatment are particularly important for those women at risk of upper genital tract complication, such as those who are infected and undergo a genital tract surgical procedure-for example, termination of pregnancy. ${ }^{1-4}$ Yet traditional screening procedures for detection of asymptomatically infected patients has necessitated a speculum examination to collect the infected cells of columnar epithelium from the endocervix of which $C$ trachomatis has tropism. Furthermore, using culture techniques it has been shown that $5-30 \%$ of infected women have only urethral colonisation, while only $50-60 \%$ have both endocervical and urethral infection. ${ }^{17}$ The new amplification assays, however, with the advantage of increased sensitivity and less invasive collection of clinical samples, have markedly simplified this approach. In the present study patient collected tampon samples or a first voided urine were shown to be equally suitable for detection of $C$ trachomatis by PCR or LCR, compared with a physician collected endocervical swab using the same detection technology. We have also previously shown a high patient compliance and acceptability to the tampon for sample collection in remote populations where lack of sophisticated services and transport delays in delivery of samples resulted in a detection rate by PCR to be almost double that for conventional methods for chlamydia diagnosis. ${ }^{13}$

Columnar cells are the cell of chlamydia tropism and infection, and we hypothesis that PCR or LCR applied to tampon and/or first catch urine samples detect shed and/or flushed cells infected with chlamydia. This is further supported by the findings of Thomas et $a l^{18}$ who assessed and found vaginal swabs collected for testing by LCR to be as sensitive for detection of $C$ trachomatis for women also positive in endocervical and urine samples.

Even with the added sensitivity of amplification assays to detect more infections, the quality of the specimen collected is still key to obtaining optimum diagnostic performance. We earlier found, in comparing tampons with endocervical scrapes for HPV DNA detection, that much more cellular DNA was obtained by the former. ${ }^{19}$ Furthermore, in comparing endocervical swab samples for culture compared with PCR detection for $C$ trachomatis, we found that $6 \%$ of swab samples were negative for $\beta$ globin, a marker of poorly collected 
specimens. ${ }^{7}$ This is further supported by Welsh et $a l^{20}$ who recently reported a study where they examined the quality of the cellular component of endocervical samples collected for $C$ trachomatis PCR detection, by also applying direct fluorescence; only $64 \%$ of specimens were considered adequate. They too found that the cellular quality of the sample significantly affected the ability to detect chlamydia. ${ }^{20}$ Therefore, even utilising technology with increased sensitivity, training of staff and patients in techniques of appropriate specimen collection is critical. For amplification assays such as PCR, the inclusion of an internal control such as $\beta$ globin ensures adequate sample collection.?

We recommend screening of $C$ trachomatis in all women presenting for termination of pregnancy. A patient collected tampon or first voided urine for detection by $\mathrm{PCR} / \mathrm{LCR}$ is appropriate and has high patient compliance.

We thank and acknowledge the clinicians from the Pregnancy Advisory Committee, Royal Women's Hospital, for collecting
the endocervical swab specimens, to Abbott Diagnostics for the endocervical swab specimens, to Abbott Diagnostics for
donating LCR kits, Pfizer for the donation of azithromycin, The donating LCR kits, Pfizer for the donation of azithromycin, The Royal Women's Hospital, Pathology Research and Education
Fund for financial assistance, and Jan Matthews for typing the Fund for fin

manuscript.
Contributors: SG designed and coordinated the study as well as Contributors: SG designed and coordinated the study as well as
collowite collated the data, in addition to following patients positive for chlamydia for treatment and review, $\mathrm{ST}$ and $\mathrm{SC}$ performed al the molecular biology, as well as writing the methods section of the manuscript and collated the results section as this related to the of molecular biology component of the study; $\mathrm{JH}$ was the research nurse who recruited patients, ensured samples were appropriately collected and transported

prompted patients for follow up visits.

1 Wein P, Kloss M, Garland SM. Postabortal pelvic sepsis in association with Chlamydia trachomatis. Aust NZ 7 Obstet Gynaecol 1990;30:347.

2 Stevenson MM, Radcliffe KW. Preventing pelvic infection after abortion. Int $\mathcal{F}$ STD AIDS 1995;6:305-12.

3 Moller BR, Ahrons S, Laurin J, et al. Pelvic infection after elective abortion associated with Chlamydia trachomatis. Obstet Gynaecol 1982;59:210-13.

4 Skjeldestad FE, Tuveng J, Solberg AG, et al. Induced abortion: Chlamydia trachomatis and post-abortal compliabortion: Chlamydia trachomatis and post-abortal complications. A cost ben
5 Centers for Disease Control and Prevention. Recommendations for the prevention and management of Chlamydia
trachomatis infections. MMWR 1993;42 (No RR-12):139.

6 Garland SM, Denham I, Jacobs D, et al. Chlamydia rachomatis: updated management guidelines. NVCA Venereol 1997;10:48-52.

7 Tabrizi SN, Lees MI, Garland SM. Comparison of polymerase chain reaction and culture techniques for detection of Chlamydia trachomatis. Mol Cell Probes 1993; detection of

8 Schachter J, Stamm WE, Quinn TC, et al. Ligase chain reaction to detect Chlamydia trachomatis infection of the cervix. F Clin Microbiol 1994;32:2540-3.

9 Van Doornum GJJ, Buimer M, Prins M, et al. Detection of Chlamydia trachomatis infection in urine samples from men and women by ligase chain reaction. $\mathcal{F}$ Clin Microbiol 1995;33:2042-7.

10 Pasternack R, Vuorinen P, Pitkajarvi T, et al. Comparison of manual Amplicor PCR, Cobas Amplicor PCR, and LCx assays for detection of Chlamydia trachomatis infection in women by using urine specimens. F Clin Microbiol 1997;35: $402-5$.

11 Tabrizi SN, Chen S, Fairley C, et al. Tampon-collected genial cells in detection of Chlamydia trachomatis by polymerase chain reaction. F Infect Dis 1993;168:796-7.

12 Paterson BA, Tabrizi SN, Garland SM, et al. The tampon test for trichomoniasis: a comparison between conventional methods and a polymerase chain reaction for Trichomonas vaginalis in women. Sex Transm Inf 1998;74:136-9.

13 Tabrizi SN, Paterson B, Fairley CK, et al. A self administered technique for the detection of sexually transmitted diseases in remote communities. Sex Transm Dis 1997;23:494-7.

14 Lees MI, Newnan DM, Garland SM. Simplified culture procedure for large scale screening Chlamydia trachomatis infections. F Clin Microbiol 1989;27:2811-14.

15 Holland SM, Gaydos CA, Quinn TC. Detection and differentiation of Chlamydia trachomatis, Chlamydia psittaci, and Chlamydia pneumoniae by DNA amplification. F Infect Dis 1990;162:984-7.

16 Garland SM, McInnes J, Gertig D. Genital Chlamydia trachomatis infection in Australia. Med f Aust 1993;159:90-6; including reply to letters to editor Med f Aust 1993;159: 639-40; Med F Aust 1994;160:46-7.

17 Paavonen J, Vesterinen E. Chlamydia trachomatis in cervicitis and urethritis in women. Scan f Infect Dis 1982; 32(suppl): 45-54.

18 Thomas BJ, Pierpoint T, Taylor-Robinson D, et al. Sensitivity of the ligase chain reaction assay for detecting Chlamydia trachomatis in vaginal swabs from women who are infected at other sites. Sex Transm Inf 1998;74:140-1.

19 Fairley CK, Chen S, Tabrizi S, et al. Tampons: a novel patient-administered method for the assessment of genital human papillomavirus infection. F Infect Dis 1992;165: 1103-6.

20 Welsh LE, Quinn TC, Gaydos CA. Influence of endocervical specimen adequacy on PCR and direct fluorescentantibody staining for detection of Chlamydia trachomatis infections. F Clin Microbiol 1997;35:3078-81. 